\title{
EXPRESSION PROFILING OF SELECTED MICRO RNAS COUPLED WITH MOLECULAR AND BIOCHEMICAL ANALYSES OF ASIATICOSIDES IN CENTELLA ASIATICA (L.)URB IN VITRO
}

\author{
JISHA S., HEMANTHAKUMAR A. S., GOURI P. R., SABU K. K.
}

Division of Biotechnology and Bioinformatics, Jawaharlal Nehru Tropical Botanic Garden and Research Institute, Thiruvananthapuram 695562, Kerala, India

Email: sabu@jntbgri.res.in

Received: 22 Jan 2019 Revised and Accepted: 29 Mar 2019

\section{ABSTRACT}

Objective: Centella asiatica (L.) Urb from Umbelliferae is a potential source of secondary metabolites having immense medicinal value. Asiaticoside is the major therapeutic compound. In the present study, Identification of a possible relationship between concentration/transcript level expression of asiaticoside and concentrations of growth hormones at different growth stages was observed. The current study includes molecular and biochemical evaluation of stress generated in C. asiatica at different time intervals in vitro.

Methods: The enhancement in auxin, cytokinin and final asiaticoside content were determined using immunoassay kits for auxin, cytokinin and HPLC analysis respectively. Transcript level expression at different growth phases was carried out using real-time RT-PCR. For isolation of stressrelated miRNAs, reverse transcription of total RNA using miScript II RT Kit PCR System was carried out as per instructions. The differential expression of five selected miRNAs was done by Real-Time RT-PCR. The analysis of stress in vitro was done by quantification of Hydrogen Peroxide $\left(\mathrm{H}_{2} \mathrm{O}_{2}\right)$, total phenolics and total antioxidants by $\mathrm{H}_{2} \mathrm{O}_{2}$ assay kit, total antioxidant assay kit and Folin Ciocalteau reagent respectively. The final asiaticoside content was determined by HPLC.

Results: Differential expression of key genes involved in asiaticoside pathway showed significantly higher transcript expression, which is in correlation with the final asiaticoside content. The enhanced expression of miRNAs and the analysis of $\mathrm{H}_{2} \mathrm{O}_{2}$, total antioxidant capacity and total phenolics are suggestive of generation of oxidative stress under controlled conditions.

Conclusion: The present study shows a direct correlation between oxidative stress and transcript/phytochemical estimation of asiaticoside content under in vitro conditions.

Keywords: Asiaticoside, Centella asiatica, In vitro, microRNA, Real-time RT-PCR

(c) 2019 The Authors. Published by Innovare Academic Sciences Pvt Ltd. This is an open access article under the CC BY license (http://creativecommons.org/licenses/by/4.0/) DOI: http://dx.doi.org/10.22159/ijpps.2019v11i5.32097

\section{INTRODUCTION}

Plants are normally subjected to different types of stresses. Stress in plants can be originated from the abiotic factor like surrounding environment or from biotic factors like living organisms. Stress can be either beneficial or detrimental. Enhancement in secondary metabolites is beneficial and it causes many physiological and biochemical changes in growth and development [1]. Plant tissue culture technology has evolved as enhancer which affects genome plasticity in plants due to alteration in environmental conditions. Various plant regulators protect against these stresses through different signalling pathways to nullify the adverse effects. Among these plant regulators, phytohormones are the major ones which manage the different biotic and abiotic stresses [2]. Recently the role of miRNAs in modulating the stress responses is also documented. It is reported that miRNAs in plants act as critical post-transcriptional regulators of gene expression which helps to react to various abiotic stresses during different growth phases [3]. miRNAs, that direct post-transcriptional repression of messenger RNAs, with diverse functions [4]. They are generally involved in the modulation of stability and/or translational potential of their mRNA targets [5]. Differential expressions of miRNAs implicated in plant growth and development have been observed in many plant species which were prone to abiotic stress conditions such as drought, salinity etc [6]. miRNAs may hold the key as potential targets for genetic manipulations to engineer abiotic stress tolerance in crop plants.

Centella asiatica L. Urb. has been used since prehistoric times and is used for various medicinal and cosmetic purposes, thus becoming an important commercial product. It is being used to improve learning and memory [7], and has nootropic effects [8], protects the brain from age-related oxidative damage [9], also promotes nerve growth and neuronal dendritic arborization [10]. The plant accumulates large quantities of pentacyclic triterpenoid saponins which include asiaticoside, madecassoside, madecassic acids, asiatic acid etc [11]. Asiaticoside is the major one and is responsible for most of its' therapeutic properties [12].

To date, there are no reports on the modulation of stress response in different growth stages of in vitro grown plants in terms of its endogenous hormone levels and antioxidant potential. It is the first report on the differential expression of selected miRNAs in $C$. asiatica grown in vitro in correlation with the differential expression of key genes involved in asiaticoside pathway and the final asiaticoside content. The stress response during in vitro conditions was also analysed.

\section{MATERIALS AND METHODS}

\section{Maintenance of plant material in vitro}

C. asiatica plants were maintained at Jawaharlal Nehru Tropical Botanical Garden and Research Institute, India under uniform conditions of growth. The surface sterilization was done in accordance with the earlier study [13]. The plants selected for the study were $3 \mathrm{w}$ old in vitro plantlets and the analyses were taken at different time intervals $(15,30$ and $45 \mathrm{~d}$ old $)$.

\section{HPLC analysis}

Preparation of samples, standard solution and HPLC procedures were followed as per the earlier study [13], with modifications. The chromatogram was recorded at $210 \mathrm{~nm}$ and the injection was repeated four times and the relative standard deviation of the area was calculated. The contents of asiaticoside and madecassoside in plant extracts were determined by comparing peak areas of plant samples with those of the standard. 


\section{Analysis of plant growth regulators}

\section{Estimation of auxin}

Immunoassay of indole-3-acetic Acid (IAA) was done by using the phytodetek IAA immunoassay kit according to the manufacturer's instructions (phyto detek assay kit, USA). In the standard curve, \% binding was plotted against the IAA concentration in pmoles $\mathrm{ml}^{-1}$ and the best fit curve on 4-cycle semi-log paper. Calculations were done as per the kit instructions.

\section{Estimation of cytokinin}

\section{Immunological quantification of cytokinins, t-ZR}

Preparation of leaf extracts and sep-pak purification was followed as described for auxin quantification. The immunoassay for quantification of cytokinins was carried out using the phytodetek t-ZR immunoassay kit (phyto detek assay kit, USA) according to the manufacturer's instructions. The intensity of colour is related to the sample t-ZR concentration by means of a standard curve. In the standard curve, \% binding was plotted against the t-ZR concentration in pmol ml-1 and the best fit curve on 4-cycle semi-log paper.

\section{Analysis of oxidative stress}

\section{Hydrogen peroxide $\left(\mathrm{H}_{2} \mathrm{O}_{2}\right)$ assay}

The measurement of $\mathrm{H}_{2} \mathrm{O}_{2}$ was done using the assay kit as per the kit instructions with modifications. Hundred $\mathrm{mg}$ of leaf tissues from different time intervals were ground and 2-50 $\mu$ l of sample in distilled water was added to each well and adjusted the volume to $50 \mu \mathrm{l}$ using the assay buffer. The OD at $570 \mathrm{~nm}$ was measured using a microplate reader (Biorad, USA).

\section{Determination of total antioxidant capacity}

$100 \mathrm{mg}$ of leaf tissues from 15, 30 and $45 \mathrm{~d}$ old in vitro cultures were ground and dissolved in $2 \mathrm{ml}$ of double distilled water. The thoroughly mixed sample was centrifuged at $5000 \mathrm{~g}$ for $5 \mathrm{~min}$ and the supernatant was taken. The method of preparation was followed as per the kit instructions. The absorbance was read at $570 \mathrm{~nm}$ using the microplate reader (Biorad, USA). Standard graph was prepared as a function of trolox concentration.

\section{Quantification of total phenols}

Gallic acid was used as the systematic equivalent, which is normally encountered in plant tissues in ester form [14]. Total phenols were determined by Folin Ciocalteau reagent [15]. A dilute extract of each plant extract $\left(5 \mu \mathrm{l}\right.$ of $50 \mathrm{mg} \mathrm{ml}^{-1}$ of the extract with $495 \mu \mathrm{l}$ of methanol) or gallic acid, the standard phenolic compound was mixed with $5 \mathrm{ml}$ of 1:10 diluted Folin Ciocalteau reagent and $5 \mathrm{ml}$ of $1 \mathrm{M}$ $\mathrm{Na}_{2} \mathrm{CO}_{3}$. The totals phenols were determined by colorimetry at 765 $\mathrm{nm}$. The standard graph was prepared using 3.125-200 $\mathrm{mg} \mathrm{l}$ ${ }^{1}$ solutions of gallic acid equivalent ( $\mathrm{mg} \mathrm{g}^{-1}$ of dry mass).

\section{Molecular analysis}

Total RNA was isolated from different tissue samples collected at different time intervals using RNA isoplus ${ }^{\circledR}$ reagent (Takara Clontech, USA) with slight modifications [16]. RNA quality was checked by bio photometer (Eppendorf International, Germany). Total RNA was analysed on a $2 \%$ agarose gel electrophoresis. Reverse transcription of total RNA using the miScript II RT kit PCR System (Qiagen, Germany) was carried out as per the instructions. The advantage of this kit is that the same cDNA can be used for the analysis of both mRNA and miRNA. Real-time RT-PCR analysis was carried out to assess the transcript accumulation of $C$. asiatica $S Q S$ and $\beta A S$, the two asiaticoside synthesis pathway genes and $C Y S$ (cycloartenol synthase), the key enzyme in phytosterol synthesis. miRNA primers (miR477e, miR156d, miR169f, miR397 and miR172) were also used for Real-Time RT-PCR. The asiaticoside and phytosterol specific primers were designed using Primer Premier Software version 3.2 (PREMIER Biosoft International, USA). Primers for miRNAs were designed using miRprimer2 software (Aalborg University, Denmark). The primer sequences are given in table 1. All primers were synthesized at Sigma, Bangalore. The reaction was set up in a final volume of $20 \mu \mathrm{l}$ containing $10 \mu \mathrm{l}$ dynamo colorFlash SYBR green qPCR Kit (Thermo Fisher Scientific, USA), $1.5 \mu \mathrm{l}$ of cDNA and $300 \mathrm{nmol}$ each of the designed primers and the conditions were: $50{ }^{\circ} \mathrm{C}$ for 2 min initially followed by $95^{\circ} \mathrm{C}$ for $10 \mathrm{~min}$ and 40 cycles of $95{ }^{\circ} \mathrm{C}$ for $15 \mathrm{~s}$ and $60^{\circ} \mathrm{C}$ for $1 \mathrm{~min}$ in a real-time RT-PCR machine (ABI 7500, Applied Biosystems, USA). The plant-specific 5.8S rRNA gene served as the control for constitutive gene expression in leaves. Comparative expression levels $(2-\Delta C t)$ were calculated using SDS software. Expression levels are relative to the level of 5.8S rRNA expression, which was constant in all RNA samples used and was set to 1 . Values are the means of three samples of two biological experiments assayed by quantitative PCR. The negative controls and the melting curve analyses carried out with each PCR reaction confirmed the absence of non-specific PCR products and primer dimers.

Table 1: List of gene-specific primers used in the study

\begin{tabular}{lll}
\hline Primer names & Forward primer & Reverse primer \\
\hline BASRT & TCCCTCAGCAGGAAACAAC & GGTACTCTCCAAGTGCCCATA \\
SQSRT & CAAATTTTCCGTGGC & GGGTTTATTTCTCCAGAAGAC \\
CYSRT & ATGCCTGGTTTGGTTATCACT & AACCCCACCACCATCTCTAT \\
$5.8 S$ rRNART & TCGATGGTTCACGGGATTC & TGAAGAACGGTAGCGAAATG \\
\hline
\end{tabular}

Table 2: List of miRNA primers used in the study

\begin{tabular}{lll}
\hline miRNA & Forward primer & Reverse primer \\
\hline miR477e & CTCTCCCTCAAGGGCTT & GGTCCAGTTTTTTTTTTTTTTTCAG \\
miR156d & GCAGTGACAGAAGAGAGTGA & TCCAGTTTTTTTTTTTTTTTGTGCT \\
miR169f & GAAGCCAAGAATGACTTGC & GGTCCAGTTTTTTTTTTTTTTTCAG \\
miR397 & GTTTGAGTGCAGCGTTG & GGTCCAGTTTTTTTTTTTTTTTCATC \\
miR172 & CGCAGGGAATCTTGATGATG & GTCCAGTTTTTTTTTTTTTTTGCAG \\
\hline
\end{tabular}

\section{Statistical analysis}

For each experiment, in vitro plants were analyzed and the experiments were repeated thrice. Analysis of data was carried out using the Graphpad Instat version 3.6 (Graphpad Software Inc., USA).

RESULTS

\section{Effect of in vitro culture in asiaticoside production}

In order to detect the effect of in vitro culture on asiaticoside production, HPLC analysis was carried out in both $C$. asiatica after a period of $45 \mathrm{~d}$. The in vivo field grown plants indicated the asiaticoside of $0.17 \mathrm{mg} \mathrm{g}^{-1}$ of dry weight in leaves, whereas the in vitro grown plant showed the maximum asiaticoside content 0.33 $\mathrm{mg} \mathrm{g}^{-1}$ of dry weight) which was statistically significant $(\mathrm{P}<0.001$, fig. 1). The chromatograms of the asiaticoside standard, field and in vitro plant leaves are provided in (Supplementary material. 1). Further experiments were conducted only in C. asiatica under in vitro conditions.

\section{In vitro induced changes in Auxin and Cytokinin content}

The auxin, Indole-3-Acetic acid (IAA) and cytokinins, trans-zeatin riboside (t-ZR) were quantified from the leaves of 15,30 and $45 \mathrm{~d}$ 
old in vitro plants. Quantitative determination of IAA by competitive ELISA showed a significant increase in auxin level ranging from 50 to 125 pmoles $\mathrm{ml}^{-1}$ in $C$. asiatica in vitro cultured plants from 15 to $45 \mathrm{~d}$ (fig. 2).

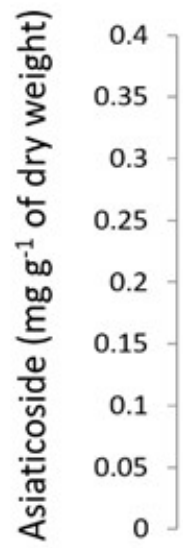

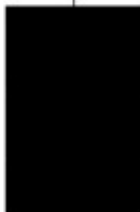

Control

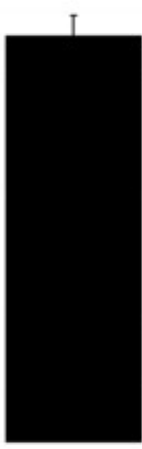

in vitro

Fig. 1: Comparison of HPLC quantitation of asiaticoside content in $C$. asiatica. The amount of asiaticoside produced by control field plants (1) and in vitro grown $C$. asiatica in the leaf tissues after a period of $45 \mathrm{~d}$. The values are mentioned in mean $\pm S D$. Number of samples in the experiment $=3$

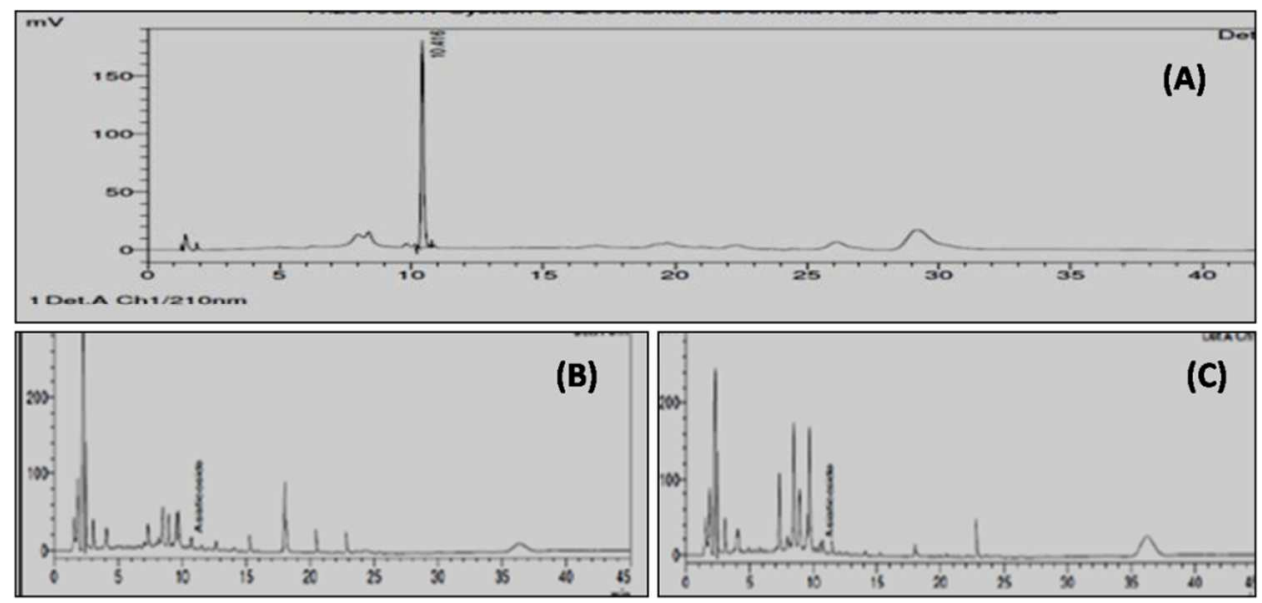

Supplementary material 1: HPLC chromatograms for the asiaticoside standard (A), in vivo (B) and in vitro (C) grown C. asiatica

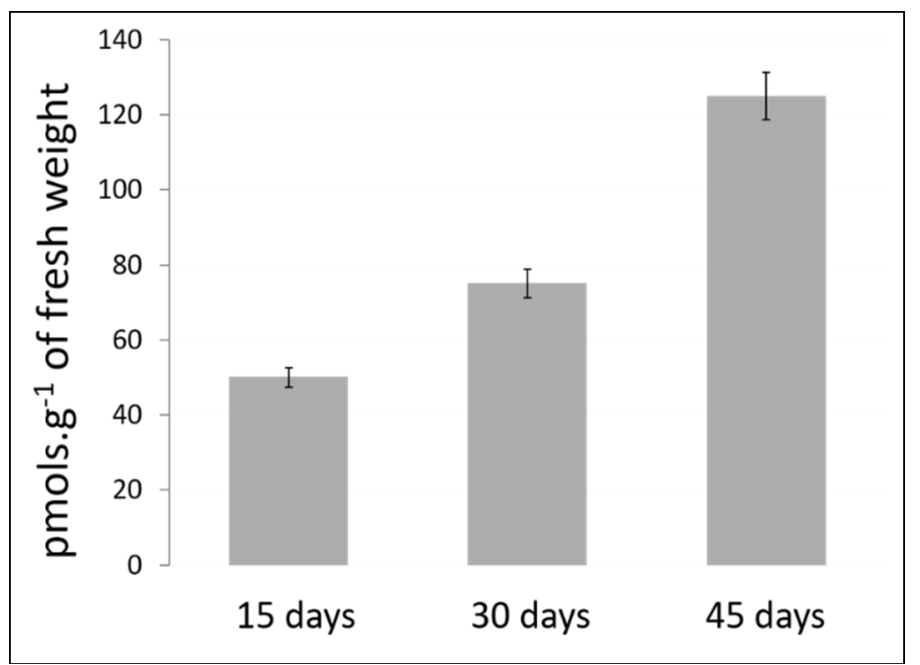

Fig. 2: Quantification of auxin in the leaves of $C$. asiatica. Estimation of the endogenous levels of hormone auxin-IAA in the leaves of in vitro grown $C$. asiatica plants at 15,30 and $45 \mathrm{~d}$ interval. The values are mentioned in mean $\pm S D$. Number of samples in the experiment=3 
The standard graph was prepared for the estimation of IAA. Similarly, the quantitative determination of trans-zeatin riboside (t-ZR) by competitive ELISA showed an augmentation in cytokinin level from 15 to $125 \mathrm{pmol} \mathrm{ml}^{-1}$ in cultured plants from 15 to $45 \mathrm{~d}$. The level of both the hormones showed a drastic enhancement
$(\mathrm{P}<0.001)$ among all the three durations tested (fig. 3$)$. The standard graph was prepared for the estimation of IAA. After $45 \mathrm{~d}$ of in vitro culture, a significant accumulation of these hormones was observed which indicates the degree of the stress-induced changes in plant architecture and growth patterns in vitro.

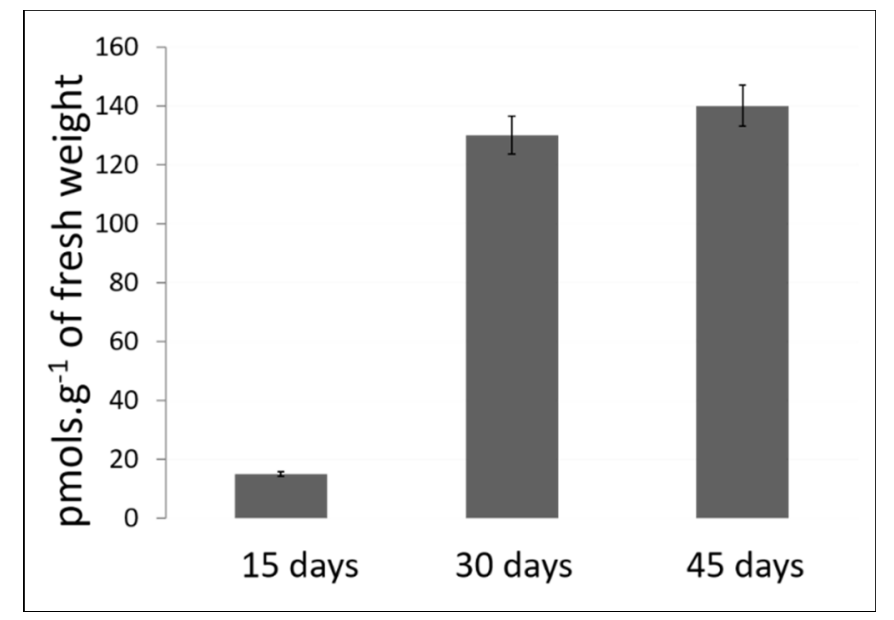

Fig. 3: Quantification of cytokinin t-ZR in the leaves of $C$. asiatica. Estimation of the endogenous levels of t-ZR in the leaves of in vitro grown $C$. asiatica plants at 15, 30 and $45 \mathrm{~d}$ interval, The values are mentioned in mean \pm SD. Number of samples in the experiment $=3$

\section{Stress-induced modulation of hydrogen peroxide, total phenolics and antioxidant capacity}

The production of $\mathrm{H}_{2} \mathrm{O}_{2}$ after $15 \mathrm{~d}$ was 100 pmol $\mathrm{ll}^{-1}$ of extract which was drastically boosted to a level of 480 pmol. $\mu^{-1}$ of extract in $45 \mathrm{~d}$ was significantly relevant $(\mathrm{P}<0.001$ fig. $4 \mathrm{a}) .30 \mathrm{~d}$ also showed a

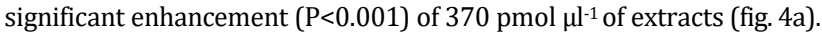
The standard graph obtained is also provided. Total antioxidant capacity of host plants was calculated after $45 \mathrm{~d}$ of in vitro culture. TAC was calculated in terms of nmol $\mu^{-1}$ of Trolox equivalent. The total antioxidant capacity of host plants was affected by in vitro cultivation, even though the change was not significant ( $P>0.05$ ) (fig. $4 \mathrm{~b})$. The antioxidant capacity showed an increase from 15-45 d (from 0.035 to 0.13 nmol. $\mu^{-1}$ of trolox equivalent respectively) with no significant change (fig. $4 \mathrm{~b}$ ). The standard graph obtained is also provided. The total phenolic content recorded in 15,30 and $45 \mathrm{~d}$ of $C$. asiatica in vitro cultures were $1.01 \pm 0.056,10.52 \pm 0.05$ and $14.4 \pm 0.06$ respectively (fig. 4c). A significant enhancement in total phenolics $(\mathrm{P}<0.001)$ was observed and maintained a significant level even after 45 d. The standard graph was prepared for the estimation of total phenols.
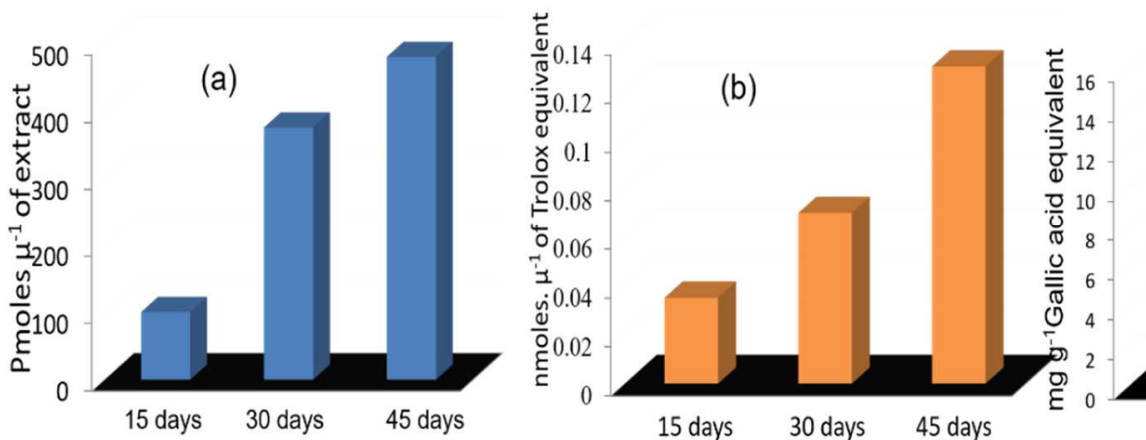

(c)

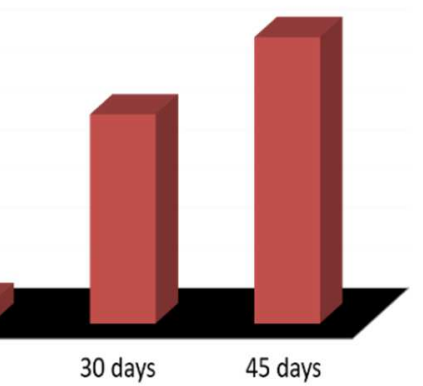

Fig. 4: Analysis of oxidative stress in C. asiatica. $\mathrm{H}_{2} \mathrm{O}_{2}$ quantification (a), Total antioxidant capacity (b) and total phenolics (c) in the leaves of $C$. asiatica in vitro cultures after 15, 30 and $45 \mathrm{~d}$. Number of samples in the experiment=3

\section{Differential expression of stress-responsive miRNAs}

Environmental stress like the limited aeration and nutrients can cause the plants to upregulate or downregulate certain miRNAs or to synthesize new miRNAs to cope with stress. For the present study, five miRNA specific primers were selected which are involved in plant stress responses directly or indirectly. The differential expression profiling was carried out using all these five miRNAs in a time-dependent manner (from $15 \mathrm{~d}$ to $45 \mathrm{~d}$ ). $5.8 S$ rRNA was selected as the internal control for the analysis of differential expression profiling in miRNAs. The relative expression level of miRNAs were calculated using $\mathrm{C}_{\mathrm{T}}$ and $2-\Delta \Delta \mathrm{CT}$ method. $2^{-\Delta \Delta \mathrm{CT}}$ or the fold change values which are greater than 1 represents upregulation of miRNA and less than 1 indicates downregulation of miRNA. Among the five primers used, significant gradual upregulations $(\mathrm{P}<0.001)$ were shown by miR477e (fig. 5a), miR156d (fig. 5b) and miR397 (fig. 5c) from 30 to $45 \mathrm{~d}$ in comparison to $15 \mathrm{~d}$ old in vitro culture. The expression profiling of miR169f and miR172 showed an entirely different pattern. An initial non-significant down regulation $(\mathrm{P}>0.05)$ in 30 $\mathrm{d}$ followed by a significant upregulation $(\mathrm{P}<0.001)$ was visualised in both miR172 (fig. 5d) and miR169f (fig. 5e). 


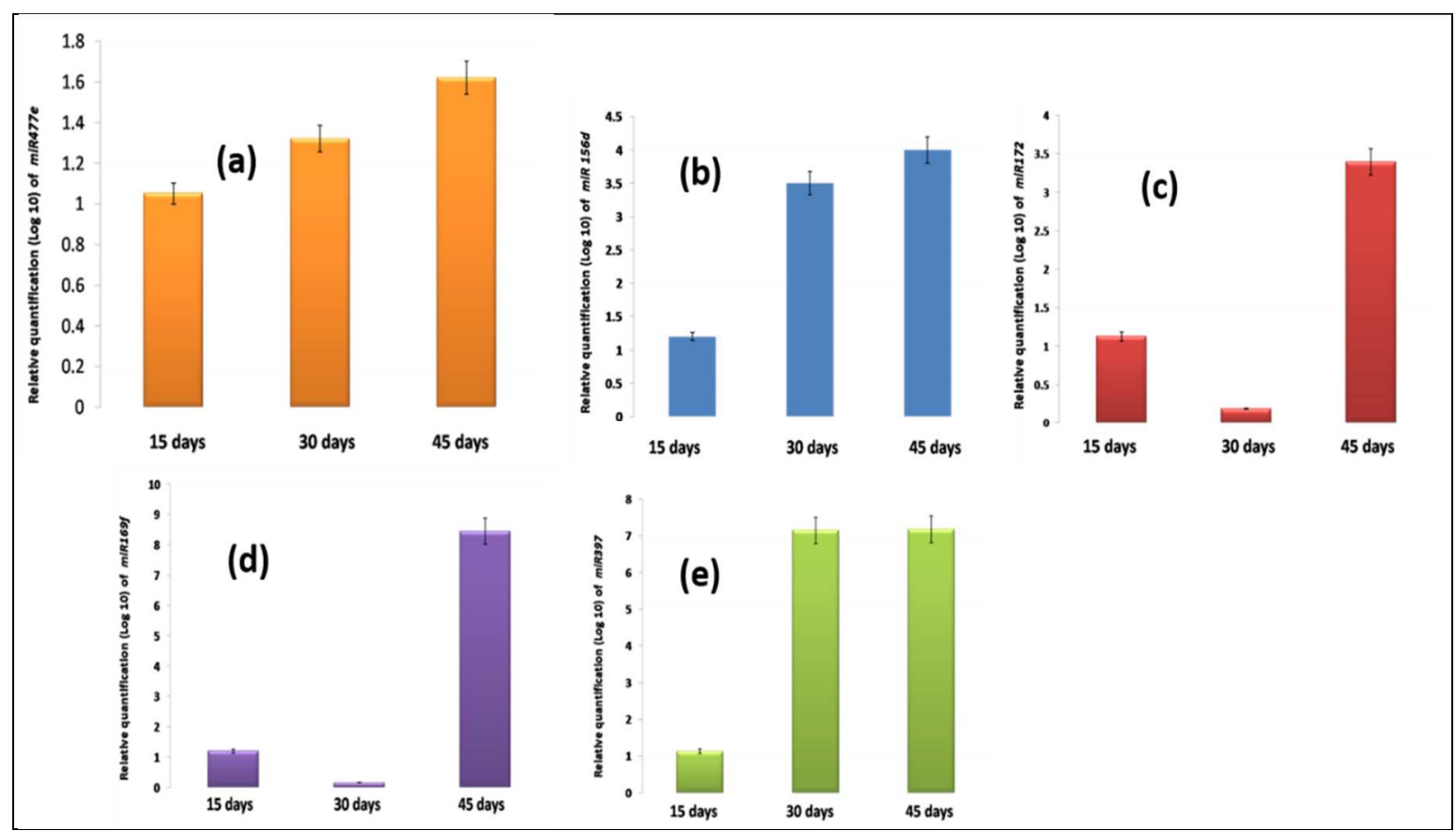

Fig. 5: Relative Quantification (RQ) by real-time RT-PCR analysis of selected miRNAs (miR477e, miR156d, miR169f, miR397 and miR172) expressed as fold expression in leaves of $C$. asiatica. Analysis was performed in leaves of $C$. asiatica in vitro. Samples were isolated at 15, 30 and $45 \mathrm{~d}$ and analysed. The values are mentioned in mean \pm SD. Number of samples in the experiment $=3$

Transcript level expression of key genes involved in an asiaticoside pathway

$\beta$ AS transcripts are strongly up regulated $(\mathrm{P}<0.001$; fig. $6 \mathrm{a})$ after a period of $30 \mathrm{~d}$ (11 times) in the leaves of $C$. asiatica over the period under controlled conditions. After $45 \mathrm{~d}$ of in vitro culture, the transcript level expression reached 16 times. A slight enhancement in the mRNA transcript level expression of $S Q S$ (P>0.05; fig. 6b) was observed over the period from 15 to $45 \mathrm{~d}$, where a slight decline in the mRNA transcript level expression of $C Y S$ ( $\mathrm{P}>0.05$; fig. 6c) was observed in the same duration. The transcript level expression was normalized with the constitutively expressing $5.8 S$ rRNA and the mean of the fold change values from the three experiments was finally taken. The transcript levels showed an increase in accumulation from 15 to $45 \mathrm{~d}(\mathrm{P}<0.001)$ of in vitro culture. The negative controls and the melting curve analyses for $\beta A S, S Q S$ and $C Y S$ carried out with each PCR reaction confirmed the absence of non-specific PCR products and primer dimers.

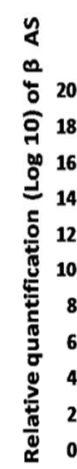

(a)

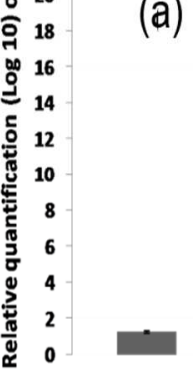

15 days

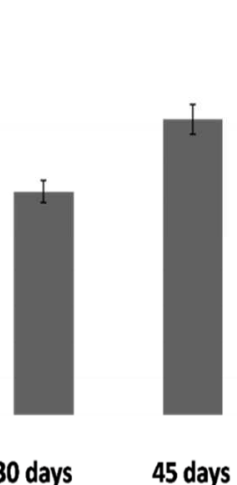

45 days

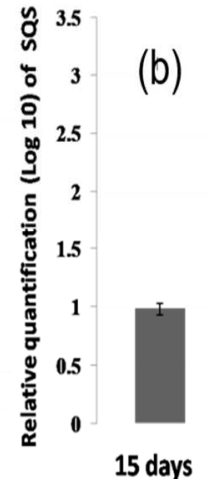

(b)

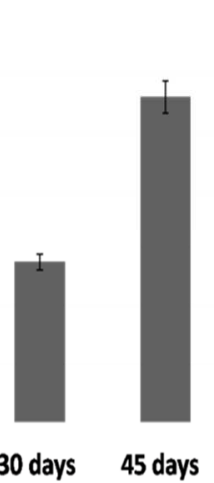

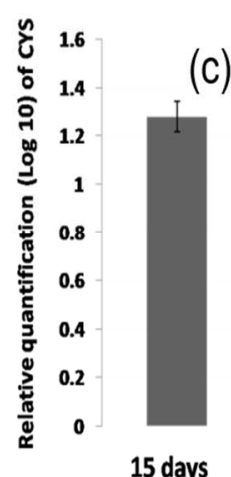

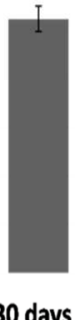

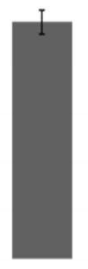

45 days

Fig. 6: Relative Quantification (RQ) by real-time RT-PCR analysis of $\beta$ Amyrin synthase (a), Squalene synthase (b) and Cycloartenol synthase (c) expressed as fold expression in the leaves of $C$. asiatica in vitro. Samples were isolated at 15, 30 and $45 \mathrm{~d}$ and analysed. The values are mentioned in mean $\pm S D$. Number of samples in the experiment $=3$

The present study explores the role of phytohormones, the powerful mediators in plant growth in $C$. asiatica under in vitro conditions. The endogenous auxin, IAA levels are higher in $45 \mathrm{~d}$ old cultures compared to 15 and 30 d old which depicts the hormone-dependent biomass enhancement in $C$. asiatica. Cytokinin is essential plant hormone that responses to external stimuli and are found in abundance in all plant species [17]. Cytokinin, t-ZR content had also been increased in vitro. In the present study, auxin acts with cytokinin in a synergistic way to mediate the stress generated in response to in vitro conditions in $C$. asiatica, which resulted in increased concentrations of major secondary metabolite. Interestingly enhancement in auxin and cytokinin content showed an identical pattern. This modulation is influenced by steady-state balance between the total antioxidants and phytohormones [18]. In view of stress-induced morphogenetic changes, total antioxidants and plant hormones play a major role in the regulatory networks because both are strongly affected by exposure to environmental stresses. Recently reports are available regarding the stress 
alleviating role of the synergistic auxin and cytokinin [19]. It is still possible that changes in hormone content were a result of the indirect effect of the environmental stress which leads to the ability of plants themselves to produce endogenous auxins and cytokinins.

Increased content of cytokinins and auxins observed in inoculated plants could be the effect of culturing the plant in a limited environment which was evidenced by the enhanced production of $\mathrm{H}_{2} \mathrm{O}_{2}$, phenolics and the total antioxidant capacity. $\mathrm{H}_{2} \mathrm{O}_{2}$, one of the most common Reactive Oxygen Species (ROS) are a fundamental fact of life in an aerobic environment [20, 21]. $\mathrm{H}_{2} \mathrm{O}_{2}$ and other ROS are produced as consequence of normal aerobic respiration, resulting from a specific process called the generation of radical superoxide or its reduction by molecules such as ascorbate, thiols and ferredoxins [22]. Recently, $\mathrm{H}_{2} \mathrm{O}_{2}$ has been regarded as a signalling molecule and regulator of the expression of some genes in cells. As one example, $\mathrm{H}_{2} \mathrm{O}_{2}$ was shown to mediate UV-B-induced gene expression as indicated by down-regulation of the UV-B-induced gene PDF1 in Arabidopsis thaliana plants exposed to a combination of antioxidants and UV-B light [23]. In vitro cultured $C$. asiatica plants at different growth stages could enhance the antioxidant property, indicated by $\mathrm{H}_{2} \mathrm{O}_{2}$ assay. A significant change in the level of $\mathrm{H}_{2} \mathrm{O}_{2}$ was observed. The significant deviation $(\mathrm{P}<0.001)$ in the generation of $\mathrm{H}_{2} \mathrm{O}_{2}$ showed at different time intervals in vitro, is indicative that it elicits a stress/defense response in the plant during the whole experimental period which in turn shows the role of stress on tissue cultured plants. $\mathrm{H}_{2} \mathrm{O}_{2}$ is thus a hallmark of ROS-mediated stress manifestation and therefore suggests a protective role under nutrient stress. Most of the medicinal plants like Hylocereus polyrhizus and Nephelium lappaceum and Syzygium zeylanicum exhibit high antioxidant potential [24-26]. A method had been designed to measure the total antioxidant status, which has been designated as total antioxidant capacity (TAC), with the advantage to measure the antioxidant capacity [27]. The TAC of $C$. asiatica at different time intervals (from 15 to $45 \mathrm{~d}$ ) shows a significant level of increase which supports the environmental stress on the plant in vitro. Exposure of plants to unfavourable environmental conditions can increase the production of ROS, which was evidenced by the enhanced total antioxidant capacity in $C$. asiatica. To protect themselves against toxic oxygen intermediates like $\mathrm{H}_{2} \mathrm{O}_{2}$, plants activate cellular antioxidant machinery. Phenolic compounds including vitamins, pigments and flavonoids have been identified to be responsible for antioxidant properties for examples catechins in tea extract [28], anthocyanins in grape seeds [29], and Eugenol in clove [30]. Reports are available on the antioxidant activities of various extracts from different parts of $C$. asiatica exhibit antioxidant activity [31]. Phenolic compounds were found out to be the major contributor of antioxidant properties [32]. According to the present study, the total phenolics enhances significantly after $45 \mathrm{~d}$ of culture. The marked effect of nutrient stress on phenolic levels is well documented [33], and has been explained in terms of increased accumulation of phenylpropanoids and lignification resulting from nutrient stress [34]. C. asiatica faces abiotic environmental stresses that have an impact on its growth and development. Conditions within the culture vessels including the presence of gelling agent, the bulk and porosity of the tissue and the temperature, also robustly influence rates of gas exchange, which is mainly driven by diffusion. The low rate of gas exchange may cause stress responses in plants which can be evidenced by the elevated levels of major plant growth regulators like auxin, cytokinin and abscisic acid. The stress under in vitro conditions can also be measured by analysis of $\mathrm{H}_{2} \mathrm{O}_{2}$, total phenolics and total antioxidant capacity.

Plants have their own defence systems to moderate the adverse effects of climatic conditions. MiRNA-mediated post-transcriptional gene regulation is one of the defence mechanisms, by targeting mRNAs for cleavage or translational repression in many eukaryotic expression systems [35]. Several prominent miRNAs are involved in biotic and abiotic stresses. Different biological experiments indicate that miRNAs play key roles during development and in response to environmental stresses [36]. Interestingly, an initial down regulation was observed in the $30 \mathrm{~d}$ of in vitro samples followed by an up regulation $(45 \mathrm{~d})$ in $m i R 169 f$ and in miR172. This significant modulation of miR169f and miR172 expression was observed at different stages in C. asiatica cultures. The miRNA expression level tended to decrease in $30 \mathrm{~d}$ and enhanced to a significant level $(\mathrm{P}<0.001)$ after $45 \mathrm{~d}$. This result has proven a deviation from the common expression pattern. A similar variation of initial down followed by up-regulation was observed in the differential expression levels of pig miRNA, hsa-miR-665 in skeletal muscle [37]. In addition, time bound expression profiles of miRNAs were also documented in rat milk whey [38]. Among the plant miRNAs, miR156 in Brassica juncea exposed to arsenic showed an initial down regulation after $24 \mathrm{~h}$ of exposure followed an upregulation at $72 \mathrm{~h}$ [39]. Tissue-specific expression of miRNAs is recorded, where the wheat miR169 was highly down regulated in leaves but upregulated in roots under drought stress, which indicates the tissue-specific modulation of miRNAs [40]. In the present study, the overexpression of miR477e, miR156d and miR397 from 15 to $45 \mathrm{~d}$ was observed from $15 \mathrm{~d}$ to $45 \mathrm{~d}$. Documented reports are available in miR156 and miR172 in regulating the development of shoot in Arabidopsis. The sequential action of these two miRNAs is involved in Arabidopsis to regulate the timing of the juvenile-to-adult conversion [41]. A significant differential expression of miR477 along with miR172 has been reported in bread wheat upon drought stress. MiR477e were reported to be highly expressed in the leaves revealed by miRNA transcriptome profiling of cassava cultivars and their wild progenitors, which are involved in the metabolic pathways [42]. Like in cassava cultivars, the $C$. asiatica under in vitro conditions also resulted in upregulation of miR477e from 15 to $45 \mathrm{~d}$ (from 2-fold to 5-fold). Upregulation of miR156d was observed under in vitro conditions. There are reports that miR156 was upregulated in A. thaliana [43] and in Triticum dicoccoides [44]. Very recent reports regarding the higher expressions of $\mathrm{miR} 156 / \mathrm{miR} 157$ in organs produced early in shoot development [45] supports our observation in this study. Besides, it is cited that the two important developmental transitions from the juvenile to reproductive phase are intervened by a collection of genes targeted by miR156, Squamosa promoter binding protein gene. In $A$. thaliana, the enhanced expression of miR397 helps to augment the plant tolerance to cold stress evidenced by the regulatory role of this miRNA in the cold pathway. The upregulation of miR397 in C. asiatica could possibly be related to the enhanced tolerance to cold stress after 15 d. MiR397 is involved in providing cold tolerance, a major environmental factor which was evidenced by the experiment conducted in $A$. thaliana. The previous studies reports overexpression of miR397 advances the cold tolerance conditions in $A$. thaliana [46]. Documented reports are available in miR156 and miR172 in regulating the development of shoot in Arabidopsis. The sequential action of the above two miRNAs are involved in Arabidopsis to regulate the timing of the juvenile-to-adult transition. The upregulation of miR156 and miR172 in C. asiatica could be correlated with the enhancement in biomass, which was supported by the enhanced levels of auxins and cytokinins. A significant differential expression of miR477 along with miR172 has been reported in bread wheat upon drought stress [40]. Like the result in cassava cultivars, the $C$. asiatica under in vitro conditions also showed upregulation in 30 and $45 \mathrm{~d}$ samples (from 2-fold to 5 -fold). On amplification with $m i R 156 d$, a significant upregulation $(\mathrm{P}<0.001)$ was observed. There are reports that miR156 was upregulated in $A$. thaliana [43], and in Triticum dicoccoides [44]. Very recent reports regarding the higher expressions of miR156/miR157 in organs produced early in shoot development [45] supports our observation in the present study. It is cited that the two important developmental transitions from the juvenile to reproductive phase are intervened by a collection of genes targeted by miR156, Squamosa promoter binding protein gene. In A. thaliana, the enhanced expression of miR397 helps to augment the plant tolerance to cold stress evidenced by its' regulatory role in the cold pathway. The upregulation of miR397 in C. asiatica could be the enhanced tolerance to cold stress after $15 \mathrm{~d}$.

The study observes the differential expression of miRNA specific primers which are directly or indirectly involved in one or other plant stress responses in different ways. MiRNA expressions can be connected with the transcript level expression of the genes involved in the asiaticoside pathway and also with the final asiaticoside content. A steady enhancement in the expression of both $\beta A S$ and $S Q S$, the key genes involved in asiaticoside pathway were observed from 15 to $45 \mathrm{~d}$, which in turn correlates with the final asiaticoside 
content after $45 \mathrm{~d}$. Enhanced level of asiaticoside content in $C$. asiatica in vitro shows the ability of in vitro conditions to elicit the generation of secondary metabolites in plants as stress response. All these results indicate the limited environmental conditions can cause abiotic stress to the plants and can also tightly influence the biosynthesis and accumulation of asiaticoside in $C$. asiatica by regulating their biosynthetic mechanism, as evidenced by both molecular and biochemical characterizations. Strong enhancement in transcript level expression of $\beta A S$ by real-time RT-PCR confirms the augmentation of asiaticoside content in C. asiatica in vitro. Comparatively lesser transcript-level expression was observed in $S Q S$. The decline in transcript level expression of CYS results in lesser accumulation of phytosterols over the period. Deterioration on transcript level expression indicates diversion of phytosterol precursors which finally channelled for the biosynthesis of most effective triterpenoid, asiaticoside.

\section{CONCLUSION}

The present study shows a direct correlation between oxidative stress and transcript/phytochemical estimation of asiaticoside content under in vitro conditions. It is evident in most of the reports that asiaticoside production is more enhanced in a growth-correlated manner, which was evidenced by the augmented cytokinin and auxin levels.

\section{ACKNOWLEDGMENT}

The authors thank Director, Jawaharlal Nehru Tropical Garden and Research Institute, India for the facilities and Indian Council of Medical Research, New Delhi for providing the Research Associate ship (Grant No. BIC/11(37)/2015). We also thank the Director, Rajiv Gandhi Centre for Biotechnology for the help extended for the completion of the study.

\section{AUTHORS CONTRIBUTIONS}

Dr KK Sabu has designed the work and finalized the manuscript. Dr Jisha $\mathrm{S}$ has carried out the work and prepared the manuscript. Dr Hemanthakumar and Gouri PR have contributions in the plant tissue culture experiments involved in the study.

\section{CONFLICT OF INTERESTS}

The authors declare that they have no conflict of interest.

\section{REFERENCES}

1. Hussain MS, Fareed S, Ansari S, Rahman MA, Ahmad IZ, Saeed M. Current approaches toward the production of secondary plant metabolites. J Pharm Bioallied Sci 2012;4:10-20.

2. Tuteja N, Gill SS, Trivedi PK, Asif MH, Nath P. Plant growth regulators and their role in stress tolerance. Plant Stress. Globan Science Books; 2010.

3. Shriram V, Kumar V, Devarumath RM, Khare TS, Wani SH. MicroRNAs as potential targets for abiotic stress tolerance in plants. Front Plant Sci 2016;7:817.

4. Ambros V. The functions of animal microRNAs. Nature 2004;431:350-5.

5. Bushati N, Cohen SM. MicroRNA functions. Annu Rev Cell Dev Biol 2007;23:175-205.

6. Anjali N, Nadiya F, Thomas J, Sabu KK. Discovery of MicroRNAs in cardamom (Elettaria cardamomum Maton) under drought stress. Hindawi Dataset Papers Sci 2017. https://doi.org/ $10.1155 / 2017 / 9507485$.

7. Zheng CJ, Qin LP. Chemical components of Centella asiatica and their bioactivities. Chin J Integr Med 2007;5:348-51.

8. Tao Y, Li JM, Li YC, Pan Y, Qun Xu, Ling-Dong K. Antidepressantlike behavioral and neurochemical effects of the citrusassociated chemical apigenin. J Life Sci 2008;82:741-51.

9. Subathra M, Shila S, Devi MA, Panneerselvam C. Emerging role of Centella asiatica in improving age-related neurological antioxidant status. Exp Gerontol 2005;40:707-15.

10. Rao MKG, Rao MS, Rao G. Centella asiatica (L.) leaf extract treatment during the growth spurt period enhances hippocampal CA3 neuronal dendritic arborization in rats. eCAM 2006;3:349-57.

11. James JT, Dubery IA. Pentacyclic triterpenoids from the medicinal herb Centella asiatica (L) Urban. Molecules 2009;14:3922-41.
12. Inamdar PK, Yeole RD, Ghogare AB, de Souza NJ. Determination of biologically active constituents in Centella asiatica. J Chromatogr 1996;746:127-30.

13. Jisha S, Anith KN, Manjula S. Induction of root colonization by Piriformospora indica leads to enhanced asiaticoside production in Centella asiatica. Mycorrhyza 2012;22:195-202.

14. Haslam E, Haworth RD, Knowles PF, Gallotannins IV. The biosynthesis of gallic acid. J Chem Soc 1961;1854-9.

15. Mc Donald S, Prenzler PD, Antolovich M, Robards K. Phenolic content and antioxidant activity of olive extracts. Food Chem 2001;73:73-84.

16. Chomczynski P, Sacchi N. Single-step method of RNA isolation by acid guanidinium thiocyanate-phenol-chloroform extraction. Anal Biochem 1987;162:156-9.

17. Pils B, Heyl A. Unraveling the evolution of cytokinin signaling. Plant Physiol 2009;151:782-91.

18. Tognetti VB, Muhlenbock P, Breusegem FV. Stress homeostasisthe redox and auxin perspective. Plant Cell Environ 2012;35:321-33.

19. Bielach A, Hrtyan M, Tognetti VB. Plants under stress: involvement of auxin and cytokinin. Int J Mol Sci 2017;18:E1427.

20. Moller IM. Plant mitochondria and oxidative stress: electron transport, NADPH turnover and metabolism of reactive oxygen species. Annu Rev Plant Physiol Plant Mol Biol 2001;52:561-91.

21. Orozco Cardenas ML, Narvaez Vasquez J, Ryan CA. Hydrogen peroxide acts as a second messenger for the induction of defence genes in tomato plants in response to wounding, system in and methyl jasmonate. Plant Cell Online 2001;13:179-91.

22. Slesak I, Libik M, Karpinska B, Karpinski S, Miszalski Z. The role of hydrogen peroxide in regulation of plant metabolism and cellular signalling in response to environmental stresses. Acta Biochimica Polonica 2007; 54:39-50.

23. A-H-Mackerness S, Surplus SL, Blake P, John CF, Buchanan Wollaston V. Ultraviolet-B induced stress and changes in gene expression in Arabidopsis thaliana: Role of signaling pathways controlled by jasmonic acid, ethelene and reactive oxygen species. Plant Cell Environ 1999;22:1413-23.

24. Wahdanimgsin S, Wahyuono S, Riyanto S, Murwanti R. Antioxidant activity of red dragon fruit peel (Hylocereus polyrhizus (F. A. C. Weber) Britton and Rose) isolates using 2,2diphenyl-1-picryl hydrazyl method. Asian J Pharm Clin Res 2018;11:124.

25. Fidrianny I, Fikayuniar L, Insanu M. Antioxidant activities of various seed extracts from four varieties of rambutan (Nephelium lappaceum) using 2,2-diphenyl-1-picryl hydrazil and 2,2'-azinobis (3-ethyl benzothiazoline-6-sulfonic acid) assays. Asian J Pharm Clin Res 2015;8:227-31.

26. Bhanu DRC, Sabu KK. Fatty acid composition of the fruits of Syzygium zeylanicum (L.) DC. var. zeylanicum. Int J Curr Pharm Res 2017;9:155-7.

27. Miller NJ, Rice-Evans C, Davies MJ, Gopinathan V, Milner A. A novel method for measuring antioxidant capacity and its application to monitoring the antioxidant status in premature neonates. Clin Sci 1993;84:407-12.

28. Kikuzaki H, Nakatani B. Antioxidant effect of some ginger constituent. J Food Sci 1993;58:1407-10.

29. Hao J, Li L, Wolf M, Xu MM, Brinsko B, Yanik M, et al. Antioxidant properties and phenolics components of grape seeds. Funct Plant Sci Biotechnol 2009;1:60-8.

30. Jirovetz L, Buchbauer G, Stoilova I, Stoyanova A, Krastanov A, Schmidt E. Chemical composition and antioxidant properties of clove leaf essential oil. J Agric Food Chem 2006;54:6303-7.

31. Hamid AA, Shah MZ, Muse R, Mohamed S. Characterisation of anti-oxidative activities of various extracts of Centella asiatica (L.) urban. Food Chem 2001;77:465-9.

32. Zainol MK, Abdul-Hamid A, Yusof S, Muse R. Antioxidative activity and total phenolic compounds of leaf, root and petiole of four accessions of Centella asiatica (L.) urban. Food Chem 2003;81:575-81.

33. Chalker Scott LS, Fuchigami. In: Low temperature stress physiology in crops. (Ed) Paul HL, CRC Press; Boca Raton, Florida: 1989. 
34. Dixon RA, Paiva N. Stress induced phenyl propanoid metabolism. Plant Cell 1995;7:1085-97.

35. Liu SR, Zhou JJ, Hu CG, Wei CL, Zhang LZ. MicroRNA-mediated gene silencing in plant defense and viral counter-defense. Front Microbiol 2017;8:1801.

36. Sunkar R. MicroRNAs with macro-effects on plant stress responses. Seminars Cell Dev Biol 2010;21:805-11.

37. Verardo LL, Nascimento CS, Silva FF, Gasparino E, Toriyama E, Barbosa AR, et al. Identification and expression levels of pig miRNAs in skeletal muscle. Livest Sci 2013;154:45-54.

38. Izumi H, Kosaka N, Shimizu T, Sekine K, Ochiya T, Takase M. Time-dependent expression profiles of microRNAs and mRNAs in rat milk whey. PLoS One 2014;9:e88843.

39. Srivastava S, Srivastava AK, Suprasanna P, D'Souza SF. Identification and profiling of arsenic stress-induced microRNAs in Brassica juncea. J Exp Bot 2013;64:303-15.

40. Akdogan G, Tufekci ED, Uranbey S, Unver. TmiRNA-based drought regulation in wheat. Funct Integr Genomics 2016;16:221-33.
41. Wu L, Zhang Q, Zhou H, Ni F, Wu X, Qi Y. Rice MicroRNA effector complexes and targets. Plant Cell 2009;21:3421-35.

42. Chen X, Xia J, Xia Z, Zhang H, Zeng C, Lu C, et al. Potential functions of microRNAs in starch metabolism and development revealed by miRNA transcriptome profiling of cassava cultivars and their wild progenitor. BMC Plant Biol 2015;15:33.

43. Ferdous J, Hussain SS, Shi B. Role of microRNAs in plant drought tolerance. Plant Biotech J 2015;13:293-305.

44. Kantar M, Lucas SJ, Budak H. miRNA expression patterns of Triticum dicoccoides in response to shock drought stress. Planta 2011;233:471-84.

45. $\mathrm{Xu} \mathrm{M,} \mathrm{Hu} \mathrm{T,} \mathrm{Zhao} \mathrm{J,} \mathrm{Park} \mathrm{MY,} \mathrm{Earley} \mathrm{KW}, \mathrm{Wu} \mathrm{G}$, et al Developmental functions of miR156-regulated Squamosa promoter binding protein-like (SPL) genes in Arabidopsis thaliana. Plos Genetics 2016;12:e1006263.

46. Dong $\mathrm{CH}$, Pei H. Over-expression of miR397 improves plant tolerance to cold stress in Arabidopsis thaliana. J Plant Biol 2014;57:209-17. 